\title{
Diabetes: conhecer para cuidar
}

\author{
Diabetes: knowing to care \\ Diabetes: saber cuidar
}

Recebido: 23/07/2021 | Revisado: 28/07/2021 | Aceito: 02/08/2021 | Publicado: 07/08/2021

Brenda Senra Duque Ramos

ORCID: https://orcid.org/0000-0002-6874-487X Universidade Federal de São João del Rei, Brasil

E-mail: brendasenra2010@gmail.com

Giovana Moreira Bordim

ORCID: https://orcid.org/0000-0003-2822-6661 Universidade Federal de São João del Rei, Brasil

E-mail: giovanamoreirab@ hotmail.com

Ana Paula Martins Inácio

ORCID: https://orcid.org/0000-0003-4576-922X Universidade Federal de São João del Rei, Brasil

E-mail:anapaulamartinsinacio@yahoo.com.br

Ludmila de Souza Conceição ORCID: https://orcid.org/0000-0003-0664-6083 Universidade Federal de São João del Rei, Brasil E-mail:lud.sc@hotmail.com

Sidney Tito Vieira Portela ORCID: https://orcid.org/0000-0002-9159-6959 Universidade Federal de São João del Rei, Brasil

E-mail: portelasidney@ hotmail.com

Renata Cláudia Muffato Reis

ORCID: https://orcid.org/0000-0003-1162-003X Universidade Federal de São João del Rei, Brasil

E-mail:renatamuffatoreis@gmail.com

Raquel Alves Costa

ORCID: https://orcid.org/0000-0003-2111-0527

Universidade Federal de São João del Rei, Brasil

E-mail:raquel.costa@ufsj.edu.br

\begin{abstract}
Resumo
Diabetes Mellitus (DM) é um dos principais desafios de saúde do século XXI, por ser uma doença silenciosa e com grande potencial de má evolução se não tratada devidamente. Diante disso, é importante que a população conheça esta patologia e tome as medidas necessárias antes do seu agravamento em prol da manutenção da saúde. De igual modo, é imprescindível que os profissionais de saúde saibam conduzir um cuidado eficiente e efetivo à população, tanto focados na prevenção e promoção em saúde, como no tratamento e manutenção da saúde. Tendo como objetivo promover grupos de discussão multiprofissional sobre cuidados com o diabetes para pacientes de Unidades Básicas de Saúde (UBS) e profissionais de duas unidades de Estratégias Saúde da Família (ESF) numa cidade do interior de Minas Gerais e verificar se os grupos de discussão promovem mudanças no comportamento do autocuidado com o DM. Os encontros contaram com 57 usuários e 10 profissionais de saúde, sendo discutido o que é diabetes, suas complicações, problemas psicológicos e alimentação do diabético. Realizou-se uma oficina e com as dúvidas levantadas, criou-se vídeos e materiais informativos. A avaliação do autocuidado do DM foi realizada pelo Questionário de atividade de autocuidado com a diabetes - QAD, mas não obtivemos um resultado significativo de adesão de participação, sendo 9 respostas iniciais e 3 após as oficinas. Como resultado observou-se uma melhoria na relação usuários e profissionais das ESFs, maior conhecimento do usuário sobre sua doença e seu autocuidado, possibilitando aos estudantes uma experiência interprofissional.
\end{abstract}

Palavras-chave: Complicações diabéticas; Doença metabólica; Práticas interdisciplinares; Autocuidado.

\begin{abstract}
Diabetes Mellitus (DM) is one of the main health challenges of the 21st century, as it is a silent disease with great potential for poor evolution if not properly treated. Therefore, it is important that the population is aware of this pathology and takes the necessary measures before it worsens in order to maintain health. Likewise, it is essential that health professionals know how to conduct efficient and effective care to the population, both focused on prevention and health promotion, as well as on the treatment and maintenance of health. Aiming to promote multiprofessional discussion groups on diabetes care for patients in Basic Health Units (UBS) and professionals from two units of Family Health Strategies (ESF) in a city in the interior of Minas Gerais and to verify if the discussion groups promote
\end{abstract}


changes in self-care behavior with DM. The meetings were attended by 57 users and 10 health professionals, discussing what diabetes is, its complications, psychological problems and diabetic diet. A workshop was held and with the doubts raised, videos and informational materials were created. The DM self-care assessment was performed using the Diabetes Self-Care Activity Questionnaire - QAD, but we did not obtain a significant result of participation adherence, with 9 initial responses and 3 after the workshops. As a result, there was an improvement in the relationship between users and professionals of the ESFs, greater knowledge of users about their disease and self-care, enabling students to have an interprofessional experience.

Keywords: Diabetic complications; Metabolic disease; Interdisciplinary placement; Self care.

\section{Resumen}

La Diabetes Mellitus (DM) es uno de los principales retos sanitarios del siglo XXI, por tratarse de una enfermedad silenciosa con gran potencial de mala evolución si no se trata adecuadamente. Por ello, es importante que la población sea consciente de esta patología y tome las medidas necesarias antes de que empeore para mantener la salud. Asimismo, es fundamental que los profesionales de la salud sepan llevar a cabo una atención eficiente y eficaz a la población, tanto enfocada a la prevención y promoción de la salud, como al tratamiento y mantenimiento de la salud. Con el objetivo de promover grupos de discusión multiprofesionales sobre atención diabética para pacientes en Unidades Básicas de Salud (UBS) y profesionales de dos unidades de Estrategias de Salud de la Familia (ESF) en una ciudad del interior de Minas Gerais y verificar si los grupos de discusión promueven cambios en la propia -Cuidado comportamiento con DM. A los encuentros asistieron 57 usuarios y 10 profesionales de la salud, en los que se debatió sobre qué es la diabetes, sus complicaciones, problemas psicológicos y dieta diabética. Se realizó un taller y ante las dudas planteadas se crearon videos y materiales informativos. La evaluación del autocuidado de la DM se realizó mediante el Cuestionario de Actividad de Autocuidado en Diabetes - QAD, pero no obtuvimos un resultado significativo de adherencia participativa, con 9 respuestas iniciales y 3 posteriores a los talleres. Como resultado, se produjo una mejora en la relación entre usuarios y profesionales de las ESF, un mayor conocimiento de los usuarios sobre su enfermedad y el autocuidado, permitiendo a los estudiantes tener una experiencia interprofesional.

Palabras clave: Complicaciones diabéticas; Enfermedad metabólica; Prácticas interdisciplinarias; Autocuidado.

\section{Introdução}

O Diabetes Mellitus (DM), corresponde a um conjunto de doenças metabólicas caracterizadas por elevados níveis séricos de glicose. A sua ocorrência se dá quando o pâncreas não é mais capaz de produzir insulina ou o organismo não é capaz de utilizar a insulina produzida, levando ao acúmulo de glicose no sangue, o que chamamos de hiperglicemia. A principal função da insulina é promover a entrada de glicose nas células do organismo para utilização em diversas atividades. (International Diabetes Federation, 2019).

De acordo com o Instituto Brasileiro de Geografia e Estatística (IBGE), o DM é um dos principais desafios de saúde deste século. Seu exacerbado e rápido crescimento fez com que triplicasse o quantitativo de seus portadores nos últimos 20 anos (IBGE, 2020).

Em 2019, o Brasil ocupou a quinta posição no ranking mundial de adultos entre 20-79 anos que possuíam DM (International Diabetes Federation, 2019). Os dados demonstram que cerca de 5 milhões de brasileiros, ou seja, 11\% da população acima de 40 anos é portadora de diabetes tipo 2. Destes casos de DM, 5,8\% do total de casos no Brasil estão, na região Sudeste (Silva, Rezende, Ferreira, Dias, Helmo \& Silveira, 2015).

Já na cidade de São João Del-Rei, Minas Gerais (MG), 13.889 pacientes foram cadastrados diabéticos de acordo com dados do DataSUS (2015), alcançando cerca de 15\% do total da população sanjoanense em 2020 (IBGE, 2020).

O processo saúde-doença associado ao DM perpassa por questões individuais, sociais e econômicas, afetando tanto os indivíduos portadores, quanto à família, o sistema de saúde e a sociedade. Dessa forma, torna-se necessário que todos conheçam tal patologia, para que consigam realizar a gestão adequada dela (Salci, Meirelles \& Silva, 2018). Por tratar-se de uma doença crônica, o DM pode trazer complicações ao paciente sem diagnóstico ou com diagnóstico tardio, causando repercussões na qualidade de vida e no desenvolvimento das atividades diárias. Entre as complicações apresentadas destacamse: retinopatia, neuropatia e nefropatia (Salci, Meirelles \& Silva, 2017). 
$\mathrm{O}$ autocuidado é descrito como a prática de atividades realizadas pelo indivíduo em seu próprio benefício, que agregam a manutenção da vida, da saúde e do bem-estar. Essas ações realizadas de maneira ativa são essenciais no cuidado do DM, uma vez que os pacientes e seus familiares são responsáveis por promover mais de 95\% do tratamento recomendado aos pacientes. Mas, na prática, nem todos os pacientes sabem como devem se cuidar. Isso pode ser visto num estudo transversal com 331 pessoas realizado no Piauí demonstrando que 53,8\% dos pacientes relatam nunca terem sido orientados quanto à importância do autocuidado com os pés e até 66,5\% não tinham conhecimento da necessidade de inspecionar os sapatos antes de colocá-los. Em média, somente $29 \%$ deles já tiveram contato com alguma orientação em relação a formas de autocuidado (Rezende Neta, Silva \& Silva, 2015).

Sabe-se que um paciente, ao realizar o autocuidado corretamente, é capaz de desacelerar a evolução da doença e, por consequência, diminuir comorbidades associadas. Isso torna mais lenta a progressão do diagnóstico da doença, o que acarreta em maior qualidade de vida (Ministério da Saúde, 2013; Oliveira, Almeida, Chagas, Salazar \& Ferreira, 2017).

Como alternativa para promover o ganho mútuo de conhecimento sobre o DM e suas complicações, o trabalho interprofissional tem ganhado cada vez mais espaço, uma vez que é possível observar uma melhor atuação dos profissionais, e uma melhor progressão da doença por parte do paciente. Um estudo realizado nos Estados Unidos demonstra que um grupo de pacientes diabéticos acompanhados por uma equipe interprofissional teve uma redução em sua hemoglobina glicada e glicose, de maneira estatisticamente significante quando comparado com o grupo controle (Nagelkerk, Thompson, Bouthillier, Tompkins, Baer, Trytko \& Groeneveld, 2018).

Levando em consideração todos os pontos levantados até aqui e após uma análise prévia da população alvo - obtida por um projeto de pesquisa realizada pelo mesmo grupo anteriormente (Dados não publicados) - foi identificado que as informações sobre o diabetes eram escassas nas ESF objetos de estudo, ainda que se tratasse de uma população com número elevado de indivíduos diagnosticado.

Uma das formas de levar informações à população é através da formação de grupos para discussão de temas em rodas. O trabalho realizado por Mota, Silva, Ahrel, Castro, Silva, Carvalho-Ferreira \& Guimarães (2016) demonstrou como a experiência realizada com grupo de diabéticos foi benéfica na diminuição da ansiedade dos participantes, tendo evoluído o grupo de grau leve para grau mínimo de acordo com a escala de Beck-BAI após 1 ano e meio de encontros.

É relatado em literatura que o conhecimento sobre doença é essencial para uma boa adesão ao tratamento, refletindo diretamente nos índices laboratoriais como hemoglobina glicada (HbA1C) e glicemia de jejum. Um estudo caso-controle realizado em Goiás demonstrou eficiência significativa nesses índices: avaliando os pacientes antes das intervenções a média de HbA1C do grupo intervenção era de 9,52\% $( \pm 1,8)$. Após os encontros, observou-se uma média de $6,64 \%( \pm 0,6)$ mostrandose uma estratégia efetiva quando comparada com os valores do grupo controle, com $\mathrm{p}<0,001$. Além desse parâmetro, o estudo também constata alteração significativa em IMC $(\mathrm{p}<0,001)$ e circunferência da cintura $(\mathrm{p}<0,001)$ (Lima, Menezes \& Peixoto, 2018).

Dessa forma, a atuação interdisciplinar e as rodas de conversas tornaram-se alternativas benéficas e eficazes, promovidas com o intuito de levar conhecimento e, consequentemente, incentivar a melhoria na qualidade de vida e uma progressão mais lenta da doença nesses pacientes crônicos. Diante desses conhecimentos, este estudo teve como objetivo promover grupos de discussão multiprofissional sobre cuidados com o diabetes para pacientes de Unidades Básicas de Saúde (UBS) e profissionais de duas unidades de Estratégias Saúde da Família (ESF) numa cidade do interior de Minas Gerais e verificar se os grupos de discussão promovem mudanças no comportamento do autocuidado com o DM. 


\section{Metodologia}

Trata-se de um relato de experiência feito a partir do projeto de extensão nomeado "Diabetes: conhecer para cuidar", submetido e aprovado ao Comitê de Ética em Pesquisa de São João del-Rei (CEPSJ) sobre Certificado de Apresentação de Apreciação Ética (CAAE) número 11695819.8.0000.5151, desenvolvido por graduandas do curso de medicina e professora do Departamento de Ciências Naturais, da Universidade Federal de São João del-Rei, com o apoio da Secretaria de Saúde da cidade e da Pró-reitoria de Extensão Universitária, foram selecionadas três ESF pelo próprio município segundo a necessidade de atenção relacionada ao tema proposto.

Com a finalidade de proporcionar um aprofundamento nos conhecimentos sobre a doença, e de formas de evitar suas as complicações aos portadores, foi organizado o estudo de acordo com o ciclo de pesquisa elaborado por Minayo \& Sanches (1993), que propõe três etapas principais: fase exploratória, trabalho de campo e análise do material adquirido.

$\mathrm{Na}$ primeira fase (exploratória) foram organizadas reuniões entre os pesquisadores para revisão bibliográfica, buscando estudos prévios acerca do trabalho em grupo para a educação em saúde de pacientes com doenças crônicas, através de pesquisa na base de dados Scielo e PubMed, com os descritores de Ciências em Saúde "diabetes mellitus", "atenção primária" e "cuidado a doenças crônicas". Com o método escolhido, foram programados seis encontros com pacientes portadores de DM cadastrados nas ESF selecionadas como cenário.

Em seguida, iniciou-se a segunda fase, o trabalho de campo. Para isso, foram realizadas duas reuniões com os enfermeiros chefes de cada ESF participante, acordando melhor dia e horário para a realização dos encontros. Nas reuniões, foram abordadas também informações acerca do número de participantes e nível de envolvimento da comunidade em atividades como a proposta. Ambos frisaram a heterogeneidade de suas áreas, a importância do trabalho multidisciplinar e interprofissional, devido à ampla quantidade de casos de descompensação da doença, e o uso de ansiolíticos por grande parte dos pacientes em função da própria DM. Os responsáveis pelas unidades, em trabalho com os Agentes Comunitários de Saúde (ACS), bem como estabelecimentos da comunidade, propuseram-se a divulgar as reuniões através da distribuição de flyers produzidos pelo projeto de extensão. Além disso, foi agendada uma participação na rádio local da cidade, no intuito de convidar a população.

Realizou-se um total de seis encontros de duração de uma hora cada em uma das 3 ESF pré-selecionadas na cidade do interior de MG. Os encontros foram realizados nos salões das ESF e programados de forma multidisciplinar, buscando fornecer o máximo de informações possíveis aos participantes, tendo a presença de um convidado externo a cada reunião. No dia da primeira atividade, o projeto foi apresentado aos participantes, sendo aplicado o Termo de Consentimento Livre e Esclarecido (TCLE) - em que estava previsto a utilização de imagens e os dados do trabalho para a apresentação em eventos científicos e publicação em periódicos e revistas de divulgação científica - e o Questionário de Atividades de Autocuidado com a Diabetes (QAD) traduzido e validado (Michels, Coral, Sakae, Damas \& Furlanetto, 2010), com o intuito de entender o impacto de nosso trabalho no autocuidado dos pacientes participantes do projeto. Nesse questionário o paciente quantifica entre 0 e 7 quantas vezes na última semana praticou o autocuidado com os pés - examinar os pés, secar entre os dedos e inspecionar os calçados antes de colocá-los -, usou as medicações corretamente, seguiu a dieta e realizou exercício físico. Essa é uma forma de avaliar a adesão ao tratamento e evidenciar como o conhecimento quanto à importância do autocuidado em doenças crônicas é essencial. (Eid, Leopoldino, Oller, Pompeo, Martins \& Gueroni, 2018).

As intervenções contaram com a participação de 57 pacientes que foram em pelo menos em 1 encontro. Para serem incluídos na avaliação acima descrita o paciente deveria ter mais de 18 anos, participar de pelo menos dois encontros e concordar em preencher o QAD ao início e ao final do projeto. Pacientes que recusassem preencher o QAD foram excluídos da análise de impacto de nosso projeto. Dessa forma, 9 pacientes preencheram algum questionário e 48 foram excluídos por algum dos critérios acima. 
Quanto à temática das reuniões, foram definidas: (1) "O que é diabetes", com a presença de uma médica de família e cardiologista; (2) "Como ter uma boa alimentação", com uma nutricionista; (3) "O exercício físico para o diabético" com uma educadora física; (4) "Diabetes e suas principais complicações", promovido pelas próprias extensionistas; (5) "Apoio psicológico aos pacientes com diabetes", com um profissional psicólogo e membro da Associação de Portadores de Diabetes de São João del-Rei. Em uma das unidades, as reuniões contaram ainda com a presença dos enfermeiros e as Agentes Comunitárias de Saúde (ACS). A organização das apresentações era livre para os convidados. Foram estruturadas palestras educacionais utilizando recursos multimídias e atividades práticas como o fornecimento de receitas e a realização de exercícios físicos. Um sexto encontro foi realizado apenas entre os extensionistas e os pacientes participantes do projeto, a fim de discutir os resultados das atividades, e produzir materiais informativos, através dos conhecimentos que julgaram mais importantes para o autocuidado. Foram fornecidos cartazes, canetas, canetas hidrográficas, lápis e borracha com os quais os indivíduos, divididos em grupos de até quatro pessoas, puderam construir a produção.

A terceira etapa, de análise do material adquirido, foi realizada através da reflexão acerca das dificuldades e potencialidades das reuniões, bem como da interpretação do nível de engajamento dos participantes. Essa etapa acontece junto com os pacientes e a equipe em um primeiro momento e em seguida somente com as pesquisadoras, a fim de levantar pontos de melhorias e finalizar a produção de materiais pactuados com a comunidade para eternizar o projeto - vídeos educativos, cartilhas e manuais sobre os temas discutidos.

Por fim, com as sugestões recolhidas dos participantes foram criados e disponibilizados materiais para que fosse possível levar a outros assistidos da ESF as informações essenciais para uma melhor condução do diagnóstico. Assim, foram elaborados vídeos ilustrativos sobre os temas abordados nos encontros e cartazes com as principais informações e dúvidas mais recorrentes. O vídeo foi divulgado em redes sociais e disponibilizado para ser assistido nas salas de espera das unidades de saúde locais, de forma que o assunto esteja sempre em pauta.

\section{Resultados}

As ações visaram incentivar o cuidado e a prevenção de complicações relacionadas à Diabetes Mellitus pelos próprios pacientes, tornando-os protagonistas do seu cuidado. Nessa perspectiva, através de encontros semanais, intencionou-se instigar nos participantes aspectos relacionados ao conhecimento prévio sobre a doença, como termos conhecidos e desconhecidos, verdades e mitos e curiosidades, além de sanar dúvidas. Essas atividades tiveram o compromisso de promover saúde, de modo a incentivar a participação dos presentes na busca por novos conhecimentos que pudessem corroborar na conduta sobre a doença.

O projeto desenvolveu-se com a criação de grupos de discussão com pacientes em ESF e aplicação de questionários, além da produção de materiais de educação em saúde como cartazes e multimídia para a população. Nesse sentido, todos os aspectos relacionados à execução do projeto, como profissionais envolvidos, locais para reuniões e comunicação à sociedade, foram previamente esclarecidos para ambas as partes, como já citado na seção de metodologia.

Para que fosse possível abordar todos os temas de relevância sobre a DM, a cada encontro foi proposto um tema para discussão e convidamos um profissional para conduzir a conversa. Nos tópicos que se seguem está descrito o desenvolvimento de cada um dos temas abordados.

\subsection{O que é Diabetes Mellitus?}

A definição do termo Diabetes Mellitus foi o tema introdutório das reuniões do projeto, sendo realizado no dia 23 de agosto de 2019, com a participação de uma médica de família e cardiologista, professora do curso de medicina da UFSJ. Assim, 
foram explicados aspectos fisiopatológicos da doença, além de esclarecidas dúvidas. Nesse encontro houve no total, a participação de 44 pessoas, as quais foram dispostas em roda, de modo a facilitar e incentivar a interação entre todos (Figura 1).

É válido ressaltar que neste primeiro momento houve um grande engajamento da população, diversas questões vieram à tona e muitas incertezas sanadas. Dentre as principais questões abordadas estava a alimentação - com dúvidas como quais alimentos poderiam ser ou não consumidos, a quantidade e horários das refeições - e aspectos relacionados à glicemia, insulina e ao jejum.

Figura 1. Grupos de intervenção sobre Diabetes realizado pelo projeto "Diabetes: conhecer para cuidar" (UFSJ) em duas ESF de São João del-Rei - MG
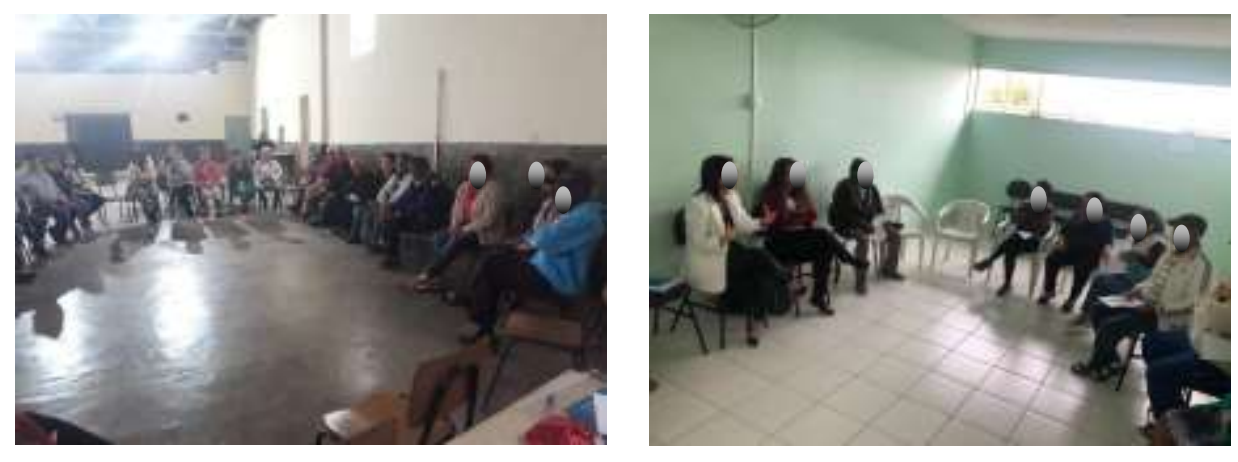

Fonte: Autores.

\subsection{Como ter uma boa alimentação?}

Como segundo tema da reunião, foi abordada a questão nutricional, tendo sido relatado posteriormente pela população participante como um dos conteúdos mais atrativos. A reunião aconteceu no dia 30 de agosto de 2019 e contou com 43 participantes. A nutricionista da Associação de Diabéticos de São João del-Rei foi a responsável por compartilhar o conhecimento técnico e científico com os presentes no que diz respeito aos melhores alimentos a serem consumidos pelos diabéticos, pré-diabéticos e não diabéticos. Para isso, foram apresentados exemplos práticos quanto aos melhores e mais acessíveis alimentos, e também realizada dinâmicas sobre fatos ou mitos na ingestão de determinados tipos de alimentos.

Neste mesmo encontro foi levado e oferecido para a população um bolo feito pelas extensionistas do projeto de receita natural e desprovida de conservantes e ou açúcares, acompanhado de cópia da receita disponibilizada, do livro Doce Vida (Vieira-Silva, Rezende, Mota, Duarte \& Teles, 2015), fruto de projeto de extensão da UFSJ. O objetivo foi exemplificar a substituição de alimentos da dieta, além de demonstrar opções interessantes e atrativas de lanches. Ao final do encontro foram sorteados dois desses livros para a comunidade.

\subsection{O exercício físico para o diabético}

Na terceira reunião do projeto, no dia 06 de setembro de 2019, foi abordada a importância do exercício físico em portadores da doença. Para isso, houve participação da educadora física do laboratório de pesquisa e intervenção psicossocial (LAPIP) da UFSJ, que abordou dúvidas referentes aos melhores tipos de exercícios físicos e horários para execução, bem como a importância da medição da taxa de glicose durante o processo. Ao todo, estavam presentes 37 pessoas.

Vale ressaltar que, nesse momento, a população já demonstrava preocupação com comorbidades e necessidades conhecidas da DM como o cuidado com os pés, visto que alguns participantes relataram ferimentos, preocupando-se com possíveis complicações, sendo dúvida frequente nas reuniões. Assim, embora esse fosse um tema específico da reunião seguinte, foi dada relevância na explicação sobre a importância do uso de calçados confortáveis - principalmente na realização de atividades físicas -, de manter os espaços interdigitais desprovidos de umidade e de sempre observar se não há ferimentos. 
Por fim, foi realizada uma sessão de alongamento, a qual poderia ser repetida por cada participante em suas respectivas residências, de modo a estimular o início ou à continuidade da prática de exercícios físicos.

\subsection{Diabetes e suas principais complicações}

Tendo em vista que diversas complicações multissistêmicas podem resultar do avanço do DM (Sanglard, Faria, Profilo, Reis, Gomes, Santiago \& Leite, 2019), mostrou-se necessário alertar os participantes sobre os principais sintomas e orientá-los sobre o valor de uma abordagem terapêutica multidisciplinar. Deste modo, o quarto encontro visou à abordagem e alerta sobre as principais complicações que podem ser desencadeadas pelo Diabetes Mellitus, e aconteceu no dia 13 de setembro de 2019, com a presença de 35 pessoas.

As complicações da diabetes foram apresentadas pelas extensionistas do projeto, abordando danos que o diabetes não controlado pode causar à diversos órgãos, a exemplo dos rins (nefropatia diabética), olhos (retinopatia diabética) e pés (neuropatia diabética).

O encontro teve boa adesão dos participantes, com diversas dúvidas e relatos de casos, os quais incluíram desde perda de visão até necessidade de amputação de dedos dos membros inferiores. $\mathrm{O}$ encontro foi finalizado com instruções de autocuidado e orientações acerca das principais medidas de prevenção às complicações.

\subsection{Apoio psicológico aos pacientes com diabetes}

A quinta reunião objetivou abordar os aspectos psicológicos dos pacientes portadores da diabetes, no intuito de auxiliar em seu enfrentamento. Assim, foi acordada a participação de um psicólogo, professor do curso de psicologia da UFSJ e ex-presidente da Associação dos Portadores de Diabetes de São João del-Rei (APD).

Sob essa ótica, houveram relatos de sentimentos como: raiva, tristeza, indignação e frustração em relação à doença; dificuldade de aceitação do diagnóstico; irritabilidade e impaciência. O momento de compartilhamentos foi essencial ao projeto, pois deu aos participantes um espaço seguro para expressarem as suas visões diante da doença, e pensar em um comportamento de esperança no combate diário à enfermidade.

\subsection{Oficina e feedback}

No sexto e último encontro foi realizada uma oficina. Por meio de cartazes e discurso aberto, os participantes puderam ponderar quais as informações que julgavam mais importantes, e como gostariam que estas estivessem disponíveis para seu acesso rápido individual em caso de dúvidas (Figura 2). Os principais tópicos solicitados foram: esclarecimento sobre como se alimentar melhor, a importância do autocuidado e a utilização correta da medicação.

A partir dessa discussão, os participantes foram convidados à elaboração de materiais informativos, os quais seriam desenvolvidos na segunda parte do projeto. Ademais, durante as reuniões os participantes relataram melhorias alimentares, além de tentativas de realização de atividades físicas. 
Figura 2. Confecção de cartazes pelos grupos de intervenção sobre Diabetes realizado pelo projeto "Diabetes: conhecer para cuidar" (UFSJ) em duas ESF de São João del-Rei - MG
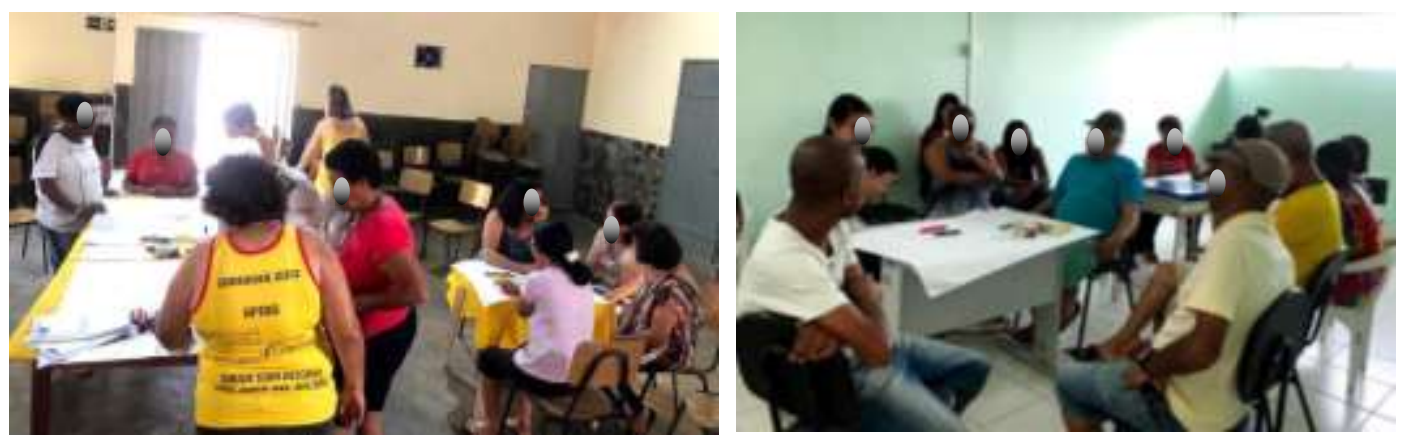

Fonte: Autores.

\subsection{O questionário de avaliação.}

Com o intuito de avaliar a eficácia dos grupos de discussão foi realizada a aplicação de um questionário de atividade de autocuidado com a diabetes - QAD (Michels et al., 2010), que já é validado na literatura. 11 pacientes preenchiam os critérios para responder os questionários, sendo 9 preenchidos de forma correta no primeiro encontro. No último encontro, somente 3 desses 9 preencheram novamente o questionário, permitindo a comparação do autocuidado com o DM antes e após os grupos de discussão.

Em relação aos resultados encontrados, antes das rodas de conversa, 33\% tinham uma alimentação inadequada, 55\% não praticavam atividade física, $66 \%$ não faziam a monitorização da glicemia, $11 \%$ não praticava o cuidado com os pés, e $100 \%$ tomava a medicação todos os dias. Nenhum participante era tabagista. Ao final do projeto $100 \%$ apresentava uma alimentação adequada, 33\% praticava atividade física, $66 \%$ faziam a monitorização da glicemia, 100\% realizavam os cuidados com os pés, e $100 \%$ utilizavam a medicação de forma adequada.

\section{Discussão}

Sabe-se que as complicações decorrentes do mal controle da DM comprometem a qualidade de vida, produtividade e sobrevida de seu portador. Ademais, o impacto econômico que tal problema gera aos cofres públicos é significativo, uma vez que, em geral, trata-se de internações hospitalares prolongadas com vários procedimentos associados. Dessa forma, o autocuidado se torna uma forma vantajosa, em todos os aspectos, de evitar desfechos desfavoráveis na evolução da DM. (Fassina, Coelho, Zinezi, Silva, Bramante \& Costa, 2018).

Sabe-se que mudanças de estilo de vida são importantes para garantir a saúde e prevenir complicações em pacientes diabéticos. Portanto, é essencial que o paciente entenda a importância de mudar e manter um estilo de vida mais saudável, além de ter boa adesão ao tratamento medicamentoso e monitorar os índices glicêmicos, como medidas gerais para garantir uma glicemia não muito elevada e evitar problemas.

Observando os encontros realizados e com os dados apontados na literatura, nota-se que um dos aspectos necessários para um bom conhecimento do diabetes e do seu tratamento é a alimentação. Estudos mostram que pode haver mudança na qualidade da alimentação após intervenções de conscientização realizadas com a população (Moura, Pena, Guimarães \& Reis, 2018). Nesse sentido, é válido considerar a importância de orientações nutricionais buscando sempre desmistificar ideias do senso comum que prejudicam a evolução desses pacientes e encorajá-los a seguirem estilos de vida mais saudáveis.

Outro tema central no incentivo ao autocuidado de indivíduos diagnosticados com DM é a prática de exercícios físicos. No caso do DM tipo 2, a realização de atividades físicas regularmente no controle glicêmico é capaz de diminuir a hemoglobina glicada e a glicemia de jejum. Um estudo transversal realizado com 56 adultos mostrou que pacientes que não 
praticam atividade física tem 1,5 vezes mais chances de ter a glicose maior que $140 \mathrm{mg} / \mathrm{dl}$ do que pacientes que praticam algum exercício rotineiramente (Miranda, Carvalho, Andrade, Coelho \& Gomes-Filho,2015).

Cabe ressaltar que a literatura cita como positivo movimentos como esse, de formação de um grupo de pessoas com doenças crônicas - como é o caso da DM, uma vez que promove o desenvolvimento de vínculo e identificação entre os participantes. Estando em grupo, esses pacientes sentem-se menos sozinhos e culpados pelo seu diagnóstico, o que auxilia tanto o tratamento da DM em si quanto em questões que surgem em consequência - como a ansiedade (Mota et al., 2016).

Esse vínculo nos grupos de discussão foi evidente neste trabalho. Na análise qualitativa dos relatos dos pacientes e também dos Agentes de saúde foi notável o quanto esses encontros foram importantes para maior compreensão do DM. Entretanto, não conseguimos verificar se houve uma mudança de estilo no autocuidado do paciente, pois houve uma baixa adesão nas respostas dos Questionários de atividade de autocuidado com a diabetes - QAD.

Outro aspecto importante observado foi a importância da escuta do paciente, no último encontro foi possível verificar que algumas informações abordadas nos grupos de discussão são relevantes para a vida diária e que gostariam de tê-las em mãos em caso de dúvidas sobre o assunto. Isso possibilitou a criação de material informativo de divulgação. Essa escuta ativa do usuário no sistema de saúde é importante e a presença dos enfermeiros e agentes de saúde nas rodas de conversa e oficina possibilitou que eles pudessem entender melhor essas dúvidas dos pacientes. O que se observa, muitas vezes, é que esse conhecimento costuma ficar limitado aos profissionais de saúde, ou ser passado inadequadamente aos pacientes. O tipo de linguagem utilizada, a forma de explicar a importância desses cuidados e a frequência de repetição das instruções têm relevância na absorção e aplicação do conteúdo compartilhado e discutido nos grupos de discussão.

Por isso, estudar sobre melhores formas de educação em saúde também é uma forma de garantir que o paciente seja também responsável pelos cuidados com a sua doença e seu tratamento, não seguindo, apenas, orientações expostas, mas não interpretadas.

Há trabalhos na literatura que demonstram que a forma mais efetiva na transmissão de informações aos pacientes ocorre pela produção de materiais impressos, sejam eles folders, cartazes, cartilhas, panfletos e livretos. Com esse tipo de material em mãos, os diabéticos podem consultá-los a qualquer momento, em casa, além de ser possível o repasse do conhecimento entre diversas pessoas, podendo a educação em saúde não decorrer apenas da relação profissional-paciente. Numa revisão bibliográfica em busca de materiais impressos voltados para a educação em saúde com o objetivo de buscar entender o propósito comunicativo desses materiais e a forma de produção deles, percebeu-se, que a maior parte desses produtos era elaborada por profissionais de saúde e que possuíam um estilo de comunicação muito unidirecional, da mesma forma como acontece muitas vezes dentro de consultórios (Freitas \& Rezende Filho, 2011)

Sabendo que as intervenções são eficientes quando ocorre a escuta ativa dos usuários e com uma abordagem interprofissional, percebemos que as intervenções ocorridas neste trabalho se mostraram produtivas para todos os envolvidos, gerando impacto nos participantes dos encontros, para os profissionais envolvidos nas equipes de saúde da família e para os graduandos e docentes participantes. Um estudo realizado a partir de um projeto de extensão interdisciplinar de Alagoas mostra que profissionais em formação que participam de ações junto às comunidades se sentem mais preparados para exercer suas funções, uma vez que tem a oportunidade de uma formação integral, ampliando o potencial de cada futuro profissional (Santana \& da Silva, 2020).

\section{Considerações Finais}

Com este projeto foi possível promover grupos de discussão multiprofissional sobre cuidados com o diabetes para pacientes de duas UBS e observar que houve uma mudança no comportamento em relação ao autocuidado após a intervenção. Ademais, foi possível estreitar o laço entre os usuários e as equipes, uma vez que elas se faziam presentes nas reuniões, além 
de garantir ao paciente um maior conhecimento sobre a sua doença, os tornando protagonistas de seu próprio cuidado. Por fim, a experiência proporcionada aos estudantes participantes foi singular, uma vez que foi permitido vivenciarem uma ação interprofissional centrada no usuário, exercendo competências comuns da interprofissionalidade como a escuta ativa e a atenção para com o paciente. Outros estudos de discussões sobre a patologia do DM devem ser feitos e esse trabalho pode servir de subsídios futuros para as o trabalho interprofissional da EFS desse município e de outros do país.

\section{Agradecimentos}

À professora orientadora pela supervisão geral e apoio na redação, edição técnica e revisão final; à Pró-Reitoria de extensão universitária (PROEX) da Universidade Federal de São João del-Rei pelo financiamento e apoio na execução do projeto; à Prefeitura municipal de São João del-Rei por permitir a entrada nos serviços de saúde para realizar a intervenção; às equipes de saúde das ESF por ajudar na divulgação e realização dos encontros; e à toda a comunidade envolvida por estar sempre presente em nossos encontros.

\section{Referências}

DataSUS, Ministério da Saúde. Sistema de informação de atenção básica. Diabetes Cadastrado por Ano segundo Município (2015). http://tabnet.datasus.gov.br/cgi/tabcgi.exe?siab/cnv/SIABSmg.def.

Eid, L. P., Leopoldino, S. A. D., Oller, G. A. S. A. D. O., Pompeo, D. A., Martins, M. A., \& Gueroni, L. P. B. (2018). Fatores relacionados às atividades de autocuidado de pacientes com diabetes mellitus tipo 2. Escola Anna Nery, 22.

Freitas, F. V.; \& Resende Filho, L.A. (2011). Modelos de comunicação e uso de impressos na educação em saúde: uma pesquisa bibliográfica. Interface. 15 (36), 243-255.

Lima, C. R. D., Menezes, I. H. C. F., \& Peixoto, M. D. R. G. (2018). Educação em saúde: avaliação de intervenção educativa com pacientes diabéticos, baseada na teoria social cognitiva. Ciência \& Educação (Bauru), 24, 141-156.

Fassina, G., Coelho, G. P., de Souza Zinezi, N., da Silva, B. A., Bramante, C. N., \& Costa, J. A. (2018). Avaliação do autocuidado em pacientes portadores do pé diabético. Revista da Faculdade de Ciências Médicas de Sorocaba, 20(4), 200-206.

Instituto Brasileiro de Geografia e Estatística (IBGE). (2020). São João del Rei. https://www.ibge.gov.br/cidades-e-estados/mg/sao-joao-del-rei.html.

International Diabetes Federation (2019). IDF Diabetes Atlas. (9a ed.), Brussels, Belgium: International Diabetes Federation.

Michels, M. J., Coral, M. H. C., Sakae, T. M., Damas, T. B., \& Furlanetto, L. M. (2010). Questionário de Atividades de Autocuidado com o Diabetes: tradução, adaptação e avaliação das propriedades psicométricas. Arquivos Brasileiros de Endocrinologia \& Metabologia, 54, 644-651.

Minayo, M. C. D. S., \& Sanches, O. (1993). Quantitativo-qualitativo: oposição ou complementaridade? Cadernos de saúde pública, 9, $237-248$.

Ministério da Saúde. (2013). Secretaria de Atenção à Saúde. Departamento de Atenção Básica. Estratégias para o cuidado da pessoa com doença crônica: Diabetes Mellitus. Brasília (DF): Ministério da Saúde. (Cadernos de Atenção Básica; 36).

Miranda, S. S., Carvalho, S., Andrade, T. M., Coelho, J. M. F., \& Gomes-Filho, I. S. (2015). Atividade física e o controle glicêmico de pacientes com diabetes mellitus tipo II. Revista Brasileira de Pesquisa em Saúde/Brazilian Journal of Health Research, 17(3), 33-40.

Mota, J. M. S., Silva, A. M. D., Abrel, T. B., Castro, M. A. D., Silva, M. V., Carvalho-Freitas, M. N., \& Guimarães, A. C. (2016). Redução de ansiedade com grupo de diabéticos: interfaces físicas e psicológicas de uma intervenção. Gerais: Revista Interinstitucional de Psicologia, 9(2), 312-323.

Moura, P. C., Pena, G. D. G. P., Guimaraes, J. B., \& Reis, J. S. (2018). Educação nutricional no tratamento do diabetes na atenção primária à saúde: vencendo barreiras. Revista de APS, 21(2).

Nagelkerk, J., Thompson, M. E., Bouthillier, M., Tompkins, A., Baer, L. J., Trytko, J., \& Groeneveld, K. (2018). Improving outcomes in adults with diabetes through an interprofessional collaborative practice program. Journal of Interprofessional Care, 32(1), 4-13.

Oliveira, M. S. N. D., Almeida, G. B. S., Chagas, D. D. N. P., Salazar, P. R., \& Ferreira, L. V. (2017). Autocuidado de idosos diagnosticados com hipertensãoarterial e/ou diabetes mellitus. Rev. enferm. UFSM, 1-14.

Rezende, D. S., Silva, A. R. V. D., \& Silva, G. R. F. D. (2015). Adesão das pessoas com diabetes mellitus ao autocuidado com os pés. Revista Brasileira de Enfermagem, 68, 111-116.

Salci, M. A., Meirelles, B. H. S., \& Silva, D. M. V. G. D. (2017). Prevenção das complicações crônicas do diabetes mellitus à luz da complexidade. Revista Brasileira de Enfermagem, 70, 996-1003. 
Research, Society and Development, v. 10, n. 10, e159101018641, 2021

(CC BY 4.0) | ISSN 2525-3409 | DOI: http://dx.doi.org/10.33448/rsd-v10i10.18641

Salci, M. A., Meirelles, B. H. S., \& Silva, D. M. G. V. D. (2018). Educação em saúde para prevenção das complicações crônicas do diabetes mellitus na atenção primáriaa. Escola Anna Nery, 22.

Sanglard, M. L., de Faria, F. C., Profilo, L. T., dos Reis, L. E. A., de Souza Gomes, R. S., Santiago, L. G., \& Leite, S. A. (2019). Diabetes Mellitus: Amputação Como Consequência De Sua Complicação. Anais do Seminário Científico do UNIFACIG, (4).

Santana, D. C, \& da Silva, M. R. (2020). A percepção de estudantes da área da saúde sobre o trabalho interdisciplinar: Experiência no projeto de extensão Sorriso de Plantão. Revista Brasileira de Extensão Universitária, 11(1), 13-24.

Silva, P. L., Rezende, M. P., Ferreira, L. A., Dias, F. A., Helmo, F. R., \& Silveira, F. C. O. (2015). Cuidados com os pés: o conhecimento de indivíduos com diabetes mellitus cadastrados no programa saúde da familia. Enfermería Global, 14(1), 38-64.

Vieira-Silva, M., Rezende, D. P., Mota, J. M. S., Duarte, N. R. F. \& Teles, I. (2015). Doce Vida: Uma publicação da UFSJ e da Associação de Portadores de Diabetes de São João Del-Rei. UFSJ/PROEX 